\title{
Recomendaciones CODEPEH 2014
}

\section{CODEPEH Recommendations}

\section{Palabras clave}

Hipoacusia (congénita, diferida), audición, cribado, infancia, audiología infantil, audioprótesis, interdisciplinariedad, familia.

\section{Key words}

Hearing loss (congenital, late-onset), hearing, screening, childhood, child audiology, hearing aids, interdisciplinarity, family.

\section{INTRODUCCIÓN}

La literatura científica más reciente señala el diagnóstico precoz de la sordera como el elemento fundamental para definir el pronóstico educativo y de inclusión del niño sordo, ya que permite aprovechar el periodo crítico de su desarrollo, que ocupa los tres o cuatro primeros años de vida. En esta edad es cuando la plasticidad cerebral es mayor y tiene lugar la adquisición de determinadas habilidades cognitivas y lingüísticas, difícilmente recuperables si se pierde la oportunidad de actuar en este periodo.

La sordera reúne todos los requisitos mínimos de las patologías que son susceptibles de cribado a través de programas de detección precoz y, además, está demostrado y reconocido que se pueden instaurar de forma precoz tratamientos capaces de evitar las consecuencias de la sordera $\mathrm{o}$, al menos, paliarlas de forma significativa (Yoshinaga-Itano, Sedey, Coulter y Mehl, I998) (YoshinagaItano, Coulter y Thomson, 2000, 200I).

Nota del editor: Este artículo ha sido publicado en Núñez, F. et al. (20I4): "Recomendaciones CODEPEH 20I4", Revista FIAPAS, octubre-diciembre 20I4, nº I 5 I, Separata.

\section{Faustino Núñez Batalla \\ $<$ fnunezb@uniovi.es>}

Presidente de la CODEPEH

\section{Carmen Jáudenes}

Casaubón

<direccion@fiapas.es>

Directora de FIAPAS

Jose Miguel Sequí Canet

Hospital de Gandía-Valencia

\section{Ana Vivanco Allende}

Hospital Universitario Central de Asturias-Oviedo

Jose Zubicaray Ugarteche

Complejo Hospitalario de NavarraPamplona

Para citar:

Núñez, F. et al. (20I 5):

"Recomendaciones CODEPEH 20I4", Revista Española de Discapacidad, 3 (I): I63-I 86 .

Doi: <http://dx.doi.org/IO.5569/23405I04.03.01.09> 


\section{Periodo crítico de desarrollo en el niño}

En el caso de los niños sordos es imprescindible aprovechar el periodo crítico de desarrollo dado que es el momento en que se sientan las bases del desarrollo comunicativo y de la adquisición del lenguaje oral, así como de la maduración de la percepción auditiva y de todas las capacidades y habilidades que de ella se derivan, incidiendo sobre los procesos de maduración neurológica.

Existe una creciente evidencia científica que demuestra que cuando la identificación e intervención se lleva a cabo no más tarde de los seis meses de edad, el niño conseguirá mejores resultados (entre 20 y 40 puntos percentiles) en su capacidad de lenguaje y comunicación: vocabulario, articulación e inteligibilidad del habla, ajuste social y comportamiento (Yoshinaga-Itano, I 995) (Yoshinaga-Itano, 2004) (Yoshinaga-Itano, Sedey, Coulter y Mehl, I998).

Por tanto, se trata de un plazo de tiempo breve e irrecuperable, en el que es necesario disponer de la información auditiva para el desarrollo del cerebro y para adquirir el lenguaje oral de forma global y automática.

Sólo así es posible que los niños y niñas sordos lleguen a interiorizar de forma natural y precoz, en el momento evolutivo que corresponde, los patrones fonológicos, los elementos lingüísticos y la estructura de la lengua oral, compartiendo con sus padres interacciones comunicativas normalizadas y espontáneas, a través de un mismo código, sin restricciones de contenido o forma. Y, en consecuencia, se evitará que la falta de audición llegue a tener un efecto permanente sobre el desarrollo del niño y, más concretamente, sobre el desarrollo del lenguaje oral y de las habilidades de aprendizaje y de comunicación que dependen de él. (Conrad, I979) (Harris y Beech, I995) (Silvestre, I998) (Torres, I998) (Alegría, I999) (Silvestre y Ramspott, 2002) (Torres y Santana, 2004) (Villalba, Ferrer y Asensi, 2005) (Torres, Moreno-Torres y Santana, 2006) (Jáudenes et al., 2007).
En base a lo descrito se justifican las diferencias altamente significativas apreciadas entre las personas sordas estimuladas tempranamente y las que han recibido esta atención específica de forma más tardía y/o inadecuada (Trinidad y Jáudenes, 20II).

Es importante insistir en la trascendencia de llevar a cabo un abordaje global, integral y coordinado, con un protocolo de actuación definido en relación con el niño sordo y con su familia, teniendo en cuenta, más allá de los aspectos clínicos y audioprotésicos ligados a la audición, todas las áreas de su desarrollo (Joint Committee on Infant Hearing, 2013).

La experiencia vivida por miles de familias en nuestro país pone de relieve que el momento del diagnóstico y la actuación inmediata en relación con la adaptación protésica y el inicio de la rehabilitación logopédica son cruciales en la vida de la persona sorda. Hoy podemos afirmar que las implicaciones de la sordera se han modificado sustancialmente gracias a la atención temprana y a la avanzada tecnología protésica, normalizando la situación educativa de los niños y niñas con sordera, su desarrollo emocional, así como las vivencias y conductas familiares (Jáudenes, 2006) (Silvestre y FIAPAS, 2008).

\section{Recomendaciones de la CODEPEH}

El Documento de Recomendaciones de la CODEPEH para 20 Io recordaba que el objetivo final de todo cribado y del tratamiento temprano de la hipoacusia congénita es la optimización de la comunicación y del desarrollo social, académico y profesional de cada niño y niña con pérdida auditiva permanente, así como facilitar el acceso precoz y natural al lenguaje oral a través de la audición, aprovechando la plasticidad cerebral de los primeros años de vida y estimulando el desarrollo comunicativo y del lenguaje del niño.

Asimismo, la CODEPEH, de acuerdo con sus recomendaciones previas, quiere insistir en la necesidad de un seguimiento continuado de la salud auditiva de la población infantil. 
A pesar de que los resultados avalan el cribado neonatal universal de la hipoacusia, dado que permite el inicio precoz del tratamiento, los programas de cribado neonatal tienen su llamado "talón de Aquiles" en la tasa de abandonos en el seguimiento de los niños que presentan resultados alterados en las pruebas realizadas al nacer. De ahí la actual discusión acerca de la necesidad de realizar un cribado a ciertas edades, que permita repescar estos casos puesto que, además, algunos trastornos auditivos en la infancia no son detectables en el cribado neonatal por no estar presentes todavía, como las hipoacusias de aparición tardía o las adquiridas, así como otras hipoacusias que, aunque presentes congénitamente, no son lo suficientemente graves como para que puedan ser detectadas en ese momento (Hyde, 2005) (Georgalas et al., 2008) (Lü et al., 20I I).

Por otra parte, existen deficiencias en la planificación, además de dificultades organizativas y de disposición de recursos en relación con estos programas de cribado, que impiden garantizar la confirmación diagnóstica y el acceso a una intervención temprana de calidad.

Estos hechos no afectan a la justificación intrínseca del cribado universal neonatal de la hipoacusia. Al contrario, lo que implican es que se tienen que llevar a cabo acciones adicionales y diseñar programas más allá del cribado neonatal para asegurar que todos los niños con una hipoacusia significativa sean detectados pronto. Por ello, muchos programas incluyen algún tipo de re-cribado dirigido a ciertos niños que presentan factores de riesgo de hipoacusia de aparición tardía o progresiva. Incluso se deberían plantear programas de cribado preescolar y escolar que ya han demostrado su utilidad (Georgalas et al., 2008), (Lü et al., 20I I).

Ahora bien, la identificación temprana de estos trastornos diferidos requiere de una especial atención y conocimientos en el estamento sanitario, así como de información y sensibilización entre los educadores, que es preciso desarrollar a través de programas de educación y estrategias de información.
Con este propósito, esta Comisión considera oportuno establecer las siguientes recomendaciones dirigidas a todos los profesionales que atienden a los niños durante su infancia.

\section{Detección precoz de la hipoacusia diferida}

\subsection{Epidemiología}

Los trastornos auditivos suponen un problema de salud muy frecuente en la población infantil. Aproximadamente tres de cada r.oo० niños nacen con pérdidas auditivas severas y profundas bilaterales. La incidencia de pérdidas moderadas y leves es mucho mayor (uno de cada Ioo). A esto se añaden las pérdidas adquiridas en el período preverbal, por lo que la prevalencia de trastornos auditivos resulta aún más elevada.

La discapacidad auditiva puede ser una alteración oculta o invisible a ojos de la mayoría, que tampoco es sencillo detectar por métodos clínicos ordinarios. Sin embargo, genera unas consecuencias que pueden llegar a ser muy graves.

Casi la mitad de todos los bebés que presentan una pérdida auditiva no tienen ninguno de los factores de riesgo conocidos para esta alteración. Este dato fundamenta el cribado universal. Cuando se estudian poblaciones seleccionadas según determinados factores clínicos de alto riesgo de daño para la audición, la incidencia de trastornos auditivos se incrementa entre Io y I4 veces (Mauk et al., I99I), siendo importante un seguimiento particular de estos pacientes. Por otra parte, no hay que olvidar que más del $95 \%$ de los padres de niños con deficiencias auditivas son normoyentes (Oysu et al., 2002) (Mitchell y Karmchmer, 2002).

La amplia implantación de los programas de cribado neonatal de la hipoacusia puede llevar a pensar que, descartada en ese momento, esta patología ya no puede afectar al niño a lo largo 
de su desarrollo. Ésta es una falsa y peligrosa creencia que puede perjudicar seriamente el futuro de esos niños puesto que factores postnatales no infrecuentes pueden originar una pérdida auditiva que comprometa alcanzar los hitos de desarrollo adecuados.

Varios estudios (Lü et al., 20I I) (Chen et al., 20I3) demuestran que hasta un $0,75^{-}-0,77$ por mil de niños preescolares presentan pérdida auditiva permanente a pesar de haber superado el despistaje neonatal. Según Watkin (Watkin y Baldwin, 20I I) (Watkin et al., 20I2) la prevalencia podría pasar del 2,52 por mil al nacer (cualquier tipo y grado de hipoacusia) al 3,64 por mil en la etapa de la educación primaria.

En amplias cohortes de niños se ha visto que, aún en los casos con altas sensibilidades en las pruebas neonatales, estas solo identifican el 56-59\% de niños en edad escolar con sordera. Viéndolo de otra forma, hasta uno de cada Io niños con hipoacusia congénita requerirá detección por revisiones postnatales a pesar de tener protocolos de cribado bien establecidos. A estos niños se añaden un $0,25^{-0,56}$ por mil que adquieren o presentan la hipoacusia en periodo postnatal y que también necesitan esas revisiones para su diagnóstico.

Por tanto, se piensa que la prevalencia de hipoacusia infantil en la etapa escolar aproximadamente duplica la esperada en la etapa neonatal, aunque otros estudios elevan esa cifra a 5 veces más (Tokgöz-Y1lmaz et al., 2013), habiendo autores que señalan que la prevalencia global de una hipoacusia de aparición tardía es del Iо \% sobre todas las hipoacusias de la infancia, pero aún podría ser mayor, llegando al $20 \%$ (Benito Orejas, 20I3) (Georgalas et al., 2008).

Todo ello sugiere la necesidad de protocolos diagnósticos que permitan identificar los casos de sordera postneonatal.

\subsection{Etiología}

Gran parte de las sorderas de inicio tardío en la infancia parecen ser debidas a defectos genéticos. Por ello, la tendencia diagnóstica actual debería ir encaminada hacia la realización de estudios genéticos con secuenciación de última generación que permite el estudio simultáneo de muchos genes involucrados en la sordera (en el momento actual hay más de I 50 loci y 64 genes) (Martins et al., 20I3).

Por tanto, se abre la puerta a la terapia génica en los pacientes con alguna mutación relacionada con sordera, lo que puede cambiar totalmente el panorama actual (Chien et al., 20I4).

En el caso de que la genética no justifique la patología auditiva habría que pensar en otras causas como infección congénita por citomegalovirus (CMV) o en trastornos del acueducto del vestíbulo, lo que obliga al estudio de la infección por CMV y a la realización de estudios de imagen complementarios (Alford et al., 20I4).

En cualquier caso hay que recordar que no sólo existen estas causas, sino que el espectro de factores de riesgo es mucho más amplio.

Se debería estar especialmente atento a niños con enfermedades o situaciones de riesgo de pérdida auditiva de posible inicio en la infancia, siguiendo esta clasificación de los tipos y causas de hipoacusia tardía (Smith y Gooi, 20I4):

- Hipoacusia transmisiva (tabla I)

- Hipoacusia neurosensorial (tabla 2)

- Hipoacusia central (tabla 3)

Entre todas estas causas destacan, por su frecuencia e importancia, las siguientes:

- Citomegalovirus: la infección por CMV congénita es la más común con una prevalencia de $0,5 \%$ en recién nacidos, de los cuales más del $94 \%$ son asintomáticos y, de éstos, un $22 \%$ desarrolla hipoacusia de forma neonatal o diferida. Alrededor de un $6 \%$ son sintomáticos y, de éstos, el $33-60 \%$ desarrolla hipoacusia. La pérdida auditiva es progresiva en el I I- $50 \%$ de los casos y de inicio tardío en el 5-1 $8 \%$ (Foulon et ál., 2008). 
Es importante su diagnóstico precoz para valorar el tratamiento farmacológico con ganciclovir o valganciclovir, dado que varios estudios han demostrado su utilidad para mejorar o impedir la progresión de la sordera en estos niños.

- Traumatismo craneoencefálico grave: las fracturas del temporal son un caso de pérdida auditiva frecuente. Las longitudinales ( $80 \%$ de frecuencia) producen pérdida auditiva conductiva por sangre en oído medio o también por perforación timpánica. Las transversas se asocian más a pérdida neurosensorial pero pueden producir lesiones en la cadena osicular. Por otro lado, traumatismos sin fractura, si son de envergadura suficiente, pueden conllevar una pérdida auditiva.

- Estancia en UCIN mayor de 5 días: todos los niños ingresados en Unidades de Cuidados Intensivos Neonatales más de 5 días, se deben considerar con riesgo elevado de hipoacusia, puesto que muchas de las patologías asociadas afectan a este grupo de pacientes (prematuridad, hiperbilirrubinemia, circulación con membrana extracorpórea-ECMO-, hipoxia perinatal, hemorragia intraventricular grados 3 y 4 , infecciones).

- Otitis serosa: la persistencia del fluido en las otitis secretoras es muy frecuente y genera pérdidas auditivas que alteran el desarrollo. Hasta un Io \% de niños presentan otitis secretora a los 3 meses de una otitis aguda. La pérdida media es de alrededor de $25 \mathrm{~dB}$.

Neuropatías auditivas genéticas: que pueden afectar a la audición como única manifestación clínica.

\subsection{RECOMENDACIONES CODEPEH en relación con la detección precoz de la hipoacusia diferida}

- Es necesario llevar a cabo una vigilancia posterior al cribado neonatal en el ámbito de la Atención Primaria.
- En cada visita periódica del Programa del "Niño Sano" se deben evaluar: las habilidades auditivas, el estatus del oído medio y los hitos del desarrollo. Es recomendable que se utilice el algoritmo propuesto por la CODEPEH para aplicarlo a los 6, I 2, I 8, 24 y 48 meses de edad (Figura I).

Si un niño no pasa la evaluación debe ser inmediatamente derivado a un ORL o a una Unidad de Hipoacusia Infantil, con el fin de ser estudiado.

- $\quad$ Es necesario un cuidadoso examen del estado del oído medio a aquellos niños en los que se compruebe una otitis serosa y, si ésta persiste durante al menos 3 meses seguidos, habrán de ser remitidos para una evaluación otológica.

- Los niños con anomalías del desarrollo y del comportamiento deben ser evaluados en la esfera auditiva al menos una vez por el ORL, prestando especial atención en este caso a las otitis serosas recidivantes o persistentes que pueden empeorar su pronóstico.

- Todos los niños con un indicador de riesgo de hipoacusia (Tabla 4 ), independientemente de los hallazgos en su seguimiento, deben ser remitidos para una evaluación audiológica, al menos una vez entre los 24 y 30 meses de edad. Aquellos niños con indicadores de riesgo muy asociados con la hipoacusia de desarrollo diferido, tales como la oxigenación extracorpórea o una infección por citomegalovirus, deben ser sometidos a evaluaciones audiológicas más frecuentes. La confirmación de una hipoacusia en un niño se considera factor de alto riesgo respecto de sus hermanos, que deben ser sometidos en este caso a una evaluación audiológica.

- Todos los niños en cuya familia exista una preocupación significativa acerca de su audición o su comunicación, con independencia de su edad, deben ser 
remitidos sin demora al ORL o a una Unidad de Hipoacusia Infantil para las pertinentes valoraciones audiológicas y del lenguaje. Habrá que proceder de igual manera si la sospecha parte del educador.

\section{PUNTOS CLAVE}

- Sensibilizar, informar y formar a los profesionales que atienden al niño desde el nacimiento y durante la primera infancia, tanto del ámbito sanitario como del educativo

- Incluir las valoraciones del desarrollo auditivo del niño en cada visita a la consulta de atención primaria, independientemente de que presenten o no factores de riesgo

- Realizar unas pruebas de cribado más exhaustivas en las cinco revisiones del "Niño Sano" ya establecidas, a los 6, I 2, I 8, 24 meses y a los 4 años

- Hacer un examen físico cuidadoso del oído y valorar la existencia de anomalías que se asocian a hipoacusia

- Atender a las observaciones hechas por la familia del niño y sus educadores, trasladar la información suficiente y las orientaciones necesarias de forma clara y concreta, involucrándoles en el proceso

\section{Valoración audiológica y adaptación protésica infantil}

El proceso de diagnóstico audiológico, así como el de habilitación auditiva y del lenguaje, tienen la misma prioridad y deben llevarse a cabo en los primeros meses de vida para maximizar el desarrollo óptimo del niño.
Ambos procesos comienzan de forma secuencial, pero se desarrollan simultáneamente.

\subsection{Valoración audiológica}

Conocer de forma fiable la pérdida auditiva del niño es el objetivo de la valoración audiológica para proceder a la adaptación audioprotésica que, no obstante, puede comenzar con estimaciones fiables de un número suficiente de frecuencias y, después, ir ajustándose en la medida en que se va disponiendo de información más precisa.

Los métodos empleados para la valoración varían en función de la edad y de la adquisición por parte del niño de diferentes habilidades y capacidades para la participación en la evaluación. De hecho, los niños requieren sucesivas visitas antes de que se pueda definir la configuración exacta, el grado y la naturaleza de la pérdida auditiva.

Los protocolos de valoración cambian con la edad. A los 6 meses es la edad en que se pasa de las pruebas electrofisiológicas, como procedimiento primario de estimación de umbrales, a métodos conductuales, que aportan resultados fiables si se siguen correctamente los procedimientos durante las sesiones de evaluación.

En el caso de que se trate de niños remitidos desde los programas de cribado neonatal de la hipoacusia, se debe proceder a una evaluación audiológica tras dos cribados fallidos. Existe una excepción a esta norma cuando los niños proceden de cuidados intensivos neonatales, en esta circunstancia, hay que realizar la prueba diagnóstica si superan los cinco días de estancia en UCIN.

A continuación, se describen brevemente las pruebas electrofisiológicas y las conductuales apropiadas para el niño muy pequeño.

- Potenciales evocados auditivos de tronco cerebral (PEATC)

Cuando se lleva a cabo una evaluación audiológica, los PEATC suelen ser la 
primera prueba que se aplica, si bien no se deben hacer de forma aislada respecto de otros test.

Esto se debe a que las respuestas a los clicks correlacionan mejor con los hallazgos audiométricos en el rango de las frecuencias altas, entre I000 y 4000 $\mathrm{Hz}$ (Coats y Martin, I977) (Gorga et al., I985) (Stapells, I989). Y, dado que los clicks contienen energía acústica de amplio rango frecuencial, las respuestas obtenidas no pueden considerarse referidas a ninguna frecuencia específica, por lo que las respuestas obtenidas por estos estímulos no pueden detectar trastornos en determinadas frecuencias. De ahí que el uso aislado de PEATC pueda, incluso, llevar a subestimar o dejar sin detectar una pérdida de audición en una determinada frecuencia o banda frecuencial, dependiendo del grado y configuración de la hipoacusia (Balfour et al., I998) (Stapells y Oates, I997).

No obstante, la predicción del audiograma mediante los potenciales evocados auditivos es posible, siempre que se obtengan dentro de las condiciones y parámetros adecuados.

La correspondencia entre los umbrales conductuales y los obtenidos mediante potenciales es buena, existiendo una diferencia en torno a los Io ó $20 \mathrm{~dB}$ (Balfour et al., I998) (Fjeredal y Lazuli, r989) (Stapells et al., I994).

Cierto es, por tanto, que los PEATC aislados no dan la suficiente información, pero son esenciales para tomar las decisiones adecuadas.

El momento óptimo para realizar esta prueba es mientras el niño duerme. Debe priorizarse la secuencia de la prueba en las distintas frecuencias debido a que el sueño puede interrumpirse inopinadamente o a que la anestesia no puede prolongarse.

Otros elementos necesarios dentro de la evaluación del niño pequeño son: la elaboración de una historia clínica, la timpanometría y las otoemisiones acústicas (OEA).

- Potenciales evocados auditivos de estado estable (PEAEE)

Esta prueba tiene la ventaja de predecir los umbrales específicos para distintas frecuencias en los pacientes cuando no se pueden obtener de forma válida y fiable, como en el caso de niños muy pequeños o en aquellos que presentan retraso en el desarrollo (Cone-Wesson et al., 2002) (Perez-Abalo et al., 200I) (Rance et al., 2005).

La ventaja de esta prueba es que es objetiva, no solo porque no precisa de la colaboración del paciente, sino también porque la presencia o ausencia de la respuesta se basa en un análisis estadístico y no en métodos de valoración visual como los PEATC. Los PEAEE con frecuencia se usan como complemento de los PEATC, y no como único test, para estimar los umbrales dado que aún no se dispone de tanta información y experiencia respecto de los mismos como en el caso de los PEATC.

Otra ventaja de los PEAEE es que permiten examinar los dos oídos simultáneamente, lo que significa un posible ahorro de tiempo respecto a los PEATC.

La generación de las respuestas de los PEAEE, se piensa que ocurre en el troncoencéfalo (Herdman et al., 2002). Se utilizan estímulos tonales modulados en amplitud y frecuencia para evocar respuestas que estiman con cierta exactitud los niveles auditivos de la vía aérea específicos para las distintas frecuencias. Para aplicaciones pediátricas, la modulación de los estímulos ocurre a una frecuencia de 80 a $100 \mathrm{~Hz}$, dado que los PEAEE con modulación de $80 \mathrm{~Hz}$ pueden ser obtenidos en niños pequeños que se encuentran dormidos (Cohen et al., I99I). 
La predicción de los umbrales utilizando los PEAEE se ha comprobado que es bastante fiable para estimar los resultados de la audiometría conductual (Aoyagi et al., I999), (Swanepoel et al., 2004). Los umbrales en adultos con audición normal o con hipoacusia se estiman con esta prueba con un error de entre Io y I $5 \mathrm{~dB}$ (Dimitrijevic et al., 2002). En niños existe menos información pero se conoce que la prueba, aunque sea capaz de detectar una pérdida auditiva en el rango severo o profundo, en general, solamente obtiene una predicción de umbrales fiable en el nivel moderado de la hipoacusia y en la audición normal (Pérez-Abalo et al., 200I).

- Timpanometría

La utilización de sondas de alta frecuencia ( $1000 \mathrm{~Hz}$ ) ofrecen resultados más fiables sobre la función del oído medio del niño menor de 4 meses (Margolis et al., 2003).

El reflejo estapedial puede ayudar mucho en la valoración audiológica del niño ya que, si está presente, es una orientación más hacia una caja timpánica que funciona con normalidad.

\section{- Audiometría conductual}

La audiometría por observación de la conducta da información acerca del tipo de respuestas que el niño produce y de su desarrollo auditivo.

Para los niños mayores de 5 o 6 meses la técnica preferida es la audiometría por refuerzo visual, que es una técnica de condicionamiento operante que permite que la respuesta por giro de la cabeza, que con frecuencia ocurre espontáneamente ante un sonido, se mantenga mediante el uso de reforzamiento visual.

Para maximizar la cantidad de información obtenida durante una visita (no todos los niños completarán la prueba de forma bilateral en una sola sesión), el orden de la presentación de los estímulos debe priorizarse con el fin de obtener información acerca del grado y configuración de la hipoacusia. Para el condicionamiento, un buen punto de partida es utilizar un estímulo verbal puesto que los niños lo encuentran más interesante que uno tonal y responden naturalmente con un giro de la cabeza, que puede ser reforzado.

La presentación de las frecuencias, alternando graves y agudas, permitirá construir un audiograma que contiene parte, si no toda, la información necesaria para predecir el umbral de la pérdida auditiva. En el caso de una hipoacusia severa-profunda, si existe falta de respuestas en las frecuencias altas, el clínico debería pasar a estimular los $500 \mathrm{~Hz}$ o frecuencias más bajas.

Es conveniente hacer pruebas de control para reducir la subjetividad y asegurar unos resultados válidos. Estos controles, en los que no se presenta estímulo auditivo, sirven para observar el giro de la cabeza al azar, cuya cuantificación permite determinar el número y porcentaje de falsos positivos en una sesión, que no debe superar el $30 \%$, pues siendo así indicaría que los datos de dicha sesión no son fiables.

Si somos capaces de condicionar al niño, su nivel madurativo y de desarrollo no influirá sobre los umbrales hallados. De este modo, los umbrales obtenidos usando la audiometría por refuerzo visual no diferirán sustancialmente de lo obtenido en adultos. Este tipo de audiometría no solo permite la valoración de los umbrales, sino que también informa de la integridad de las vías auditivas y de la habilidad de los niños para detectar y discriminar una estimulación auditiva.

Audiometría lúdica es un término utilizado para describir una técnica en la que se emplea el juego para obtener los umbrales. 
Puede usarse a partir de los 24 meses de edad pero está más indicada a los dos años y medio o a los tres. Esta audiometría involucra el condicionamiento del niño para responder a sonidos utilizando una actividad atractiva para él. El condicionamiento se consigue tras mostrar al niño cuatro o cinco respuestas guiadas o demostraciones. A través de esta técnica se puede obtener información sobre los umbrales en las distintas frecuencias de cada oído mediante estimulación aérea y ósea.

En el caso de niños muy pequeños, o que no consigan concentrarse en la tarea, la secuencia de frecuencias debería perseguir la obtención de la información precisa para deducir la configuración y el grado de la pérdida. Puede ser preferible conseguir una información frecuencial parcial de ambos oídos a llegar a obtenerla completa solamente de uno de ellos.

La audiometría convencional puede ser usada cuando el niño alcanza los 5 o 6 años de edad. La respuesta es típicamente la misma que la utilizada para los adultos, enseñando al niño a levantar la mano en cuanto oye el estímulo. Como en todas las técnicas conductuales, la edad cronológica no es determinante en la prueba, sino que lo es el nivel de desarrollo del niño.

En resumen, los hallazgos audiológicos conductuales pueden ofrecer unos resultados fiables, pero se debe tener cuidado en eliminar los falsos positivos utilizando pruebas control para observar el comportamiento del niño cuando no se le presenta estímulo. También se debe prestar atención a las pistas que puedan ofrecer al niño los adultos presentes en la prueba, además de evitar patrones de presentación con pistas o la subjetividad por parte del examinador.

\subsection{Adaptación protésica infantil}

Está demostrado que toda hipoacusia provoca modificaciones en el sistema auditivo central y que una adaptación protésica temprana hará que reviertan dichas lesiones. Este fenómeno es conocido como "aclimatación auditiva" (Arlinger et al., I996).

Durante años ha existido controversia en cuanto al tipo de adaptación, discutiéndose si debería ser monoaural o biaural. Aún mayores discrepancias había en la indicación de la adaptación en las hipoacusias unilaterales. Hoy día está ampliamente documentada la necesidad de la adaptación bilateral, recomendándose también la amplificación auditiva en las hipoacusias monoaurales, independientemente de su nivel, aunque en las severas se deba individualizar dependiendo de cada situación (Gelfand SA. et al.,I987).

Las prótesis auditivas pueden ser de conducción aérea u ósea, implantables (implantes de oído medio, implantes cocleares o de tronco cerebral) o externas (audífonos).

La decisión de proceder a una adaptación protésica debe estar basada en datos audiológicos, del desarrollo del lenguaje y del entorno familiar, escolar y social del niño.

Se hace hincapié en la necesidad de evaluar la audición funcional en los niños pequeños y no depender de los umbrales de la audición. Estos datos también pueden contribuir al proceso de toma de decisiones en la selección de un dispositivo para los niños con pérdida auditiva (Ben-Itzhak et al., 20I4).

Toda adaptación protésica en niños debe ir acompañada de un tratamiento logopédico adecuado y sostenido en el tiempo, así como de su inclusión educativa.

- Intervención interdisciplinar

El especialista en otorrinolaringología es el encargado de coordinar todas las actuaciones del numeroso equipo interdisciplinar que se precisa para el correcto diagnóstico y tratamiento de los niños con hipoacusia, y es el único que 
puede indicar el tratamiento audioprotésico o quirúrgico que precisen (Real Decreto 4I4/I996, de I de marzo, por el que se regula los productos sanitarios). Además es cada vez más necesario, por las características específicas de los niños, que este papel sea realizado por un Otorrinolaringólogo (ORL) que desarrolle su trabajo como ORL Infantil y con amplia formación en audiología.

Los establecimientos de audioprótesis son considerados como establecimientos sanitarios y, como tales, de cumplir con los requisitos (local, profesionales, recursos...) que exige la normativa que los regula y contar con material para hacer el diagnóstico audiométrico específico infantil, como una cabina insonorizada y juguetes calibrados. Entre sus funciones principales están: la interpretación de las prescripciones audioprotésicas, determinar las características anatomofisiológicas del oído, realizar audiometrías y todas las actuaciones necesarias para la adaptación, como la toma de la impresión de los moldes y el control y seguimiento de la adaptación protésica.

Los resultados y respuestas del niño percibidas durante el proceso de adaptación protésica se deben contrastar con la evaluación pedagógica y logopédica llevada a cabo por los logopedas y educadores del niño.

Fundamentalmente el programa de adaptación y seguimiento se desarrolla en tres ámbitos: médico (ORL Infantil), audioprotésico y logopédico.

El médico otorrinolaringólogo controlará la patología del oído medio, que es muy frecuente en los niños, para su rápida solución y evitar así la interferencia con la hipoacusia neurosensorial. Llevará a cabo un control evolutivo de la hipoacusia con sus pruebas pertinentes según la edad del niño y comprobará la calidad, efectividad y eficiencia de la adaptación protésica y su repercusión en la evolución del lenguaje e inclusión en el entorno.
El audioprotesista comprobará el correcto funcionamiento electroacústico de la prótesis, verificará el uso adecuado del audífono, comprobando la humedad, golpes, estado de la pila, etc. También valorará el correcto sellado del sistema audífono, molde, canal y sustituirá los elementos necesarios como codos, tubos y moldes. Por último, comprobará los parámetros establecidos con los objetivos y resultados obtenidos.

El logopeda será el encargado de realizar la (re)habilitación auditiva y del lenguaje. Colaborará, junto con el ORL Infantil y el audioprotesista, en la valoración de la pérdida auditiva y dará información para mejorar la adaptación.

Involucrar a los padres en el proceso del tratamiento de los niños con déficit auditivo resulta esencial, para ello se les debe facilitar información amplia, comprensible y veraz, que ajuste las expectativas sobre el pronóstico.

- Indicaciones para la adaptación audioprotésica en niños

Los niños tienen una serie de peculiaridades que hacen que la adaptación no sea fácil, siendo más complicada cuanto más pequeños sean.

Actualmente, con los programas de detección precoz de la hipoacusia, se llega a un diagnóstico certero de hipoacusia a edades muy tempranas por lo que el audioprotesista se enfrenta a unos conductos auditivos muy pequeños y a niños que no son capaces de indicar si perciben o no sonido, por lo que habrá que orientarse mediante la observación en sus reacciones y pruebas fisiológicas (Kerkhofs, 2013).

En una pérdida auditiva neurosensorial bilateral, con umbrales mayores a $25 \mathrm{~dB}$ HL, en el rango de frecuencias críticas para la discriminación del habla (ı000-4000 
$\mathrm{Hz}$ ), en todo caso se debería considerar proporcionar amplificación debido a las implicaciones que tiene para el desarrollo del lenguaje. De forma indiscutible se admite que está indicada cuando la pérdida supera los $35 \mathrm{~dB}$ HL (Tomblin et al., 20I4). Si el niño tiene pérdida con una caída brusca a partir de $2000 \mathrm{~Hz}$, o en una hipoacusia con audiograma en U, la amplificación debería valorarse individualmente según cada situación. En el caso de las perdidas profundas, se recomienda una adaptación durante al menos tres meses para, en caso de no presentar una respuesta adecuada, proceder a indicar un implante coclear.

La hipoacusia unilateral, aunque sea leve, produce problemas en la integración binaural del mensaje y en la discriminación en ambientes ruidosos, así como en la adecuada localización del sonido (Lieu et al., 2013). Actualmente está indicada la adaptación en estos casos ya que la amplificación en ese oído podría ser beneficiosa.

Se recomienda el uso de audífonos durante un período de prueba con un seguimiento durante los primeros años de vida (Briggs et al., 20I I) (Christensen et al., 20IO).

Se debería realizar una adaptación binaural siempre, salvo que se observase en el niño conductas que hicieran pensar que la adaptación en el peor oído produce un perjuicio en su rendimiento global.

- Cómo llevar a cabo la adaptación audioprotésica

Como ya se ha dicho, antes de proceder a la adaptación audioprotésica se debe hacer una serie de pruebas audiológicas objetivas por métodos fisiológicos y otras subjetivas adaptadas a la edad del paciente.

En niños mayores sería deseable intentar descubrir las zonas muertas de la cóclea por medio de pruebas como Psychophysical Tuning Curve (PTC) y Threshold Equalising Noise (TEN) (Moore et al., 2000).

Los tres pasos que deben ser implementados en la adaptación audioprotésica en la infancia son: selección, verificación y validación.

\section{a. Selección}

La respuesta a la adaptación protésica está condicionada por la patología auditiva, por el tipo de pérdida y por otras alteraciones neurológicas, y no tanto por la intensidad de ésta. La prótesis correctamente seleccionada es la que brinda la mejor amplificación teniendo en cuenta la audición residual del niño.

Hay que saber que la prótesis auditiva no va a suprimir la lesión del oído, solamente modifica el sonido para que pueda ser percibido y mejor aprovechado. El daño coclear implica no solo una pérdida de intensidad, sino fundamentalmente otras alteraciones que repercuten en la distorsión del sonido percibido.

Es importante individualizar cada caso ya que, entre otros motivos, puede haber niños en los que un mal rendimiento audioprotésico se pueda explicar por la presencia de una neuropatía auditiva o que presente lesiones centrales de las áreas de la audición.

Los audífonos deben tener una ganancia suficiente, una curva de respuesta adecuada para cualquier tipo de perdida audiométrica y un límite de salida máxima para evitar lesiones por exceso de volumen.

Hasta los IO-I 2 años de edad el audífono convencional debe ser retroauricular y debemos disponer de distintos tipos de moldes para conseguir una correcta adaptación del molde al conducto auditivo externo, sobre todo en lactantes.

En los problemas de oído medio como agenesias o lesiones supurativas crónicas 
suele ser necesaria la adaptación por vía ósea (Felton et al., 2014) (Fuchsmann et al., 2010).

En los lactantes, que suelen gatear, los micrófonos direccionales no son la mejor elección, ya que no estarán en la posición adecuada para recibir el sonido en muchos momentos del día, prefiriéndose los omnidireccionales.

El diseño de los moldes en los niños es también de gran importancia. Hay que ser muy cuidadoso ya que sus características físico-acústicas variarán según la pérdida auditiva, el volumen del conducto auditivo externo y la presión de salida del audífono. Por todo ello, deben de ser reemplazados cuando cambien estos parámetros. En los niños pequeños hay que valorar cambiarlos aproximadamente cada 4-6 meses y, a partir de los 5 años, una vez al año.

Para evitar extravíos es importante el uso de dispositivos para sujetar las prótesis auditivas dado el tamaño del pabellón y la gran movilidad del niño.

\section{b. Verificación}

El programa de adaptación a las prótesis normalmente se establece por el audioprotesista, no obstante debe estar consensuado con el especialista ORL, dado que el seguimiento concierne a ambos, así como contrastado con el logopeda. Las referencias básicas se encuentran en Northern y Downs (2002), quienes siguen el criterio de "menos tiempo de uso a más tiempo, de menos exposición al sonido a más exposición y de menos complejidad de los sonidos a más complejidad".

En la adaptación de los niños pequeños siempre hay que ser un poco conservador, ya que no tendremos en fases tempranas la información adecuada de los umbrales de disconfort del reclutamiento y otros factores, que se irán descubriendo según va creciendo el niño, lo cual obliga a ir adecuando la adaptación a todo ello de forma progresiva.
Los periodos de seguimiento deben ser:

- los primeros I 8 meses de vida, continuos a demanda de cada situación

- de los I 8 meses a los 3 años, cada 3 meses

- de los 3 a los 6 años, cada 6 meses

- mayores de 6 años con sorderas estables, cada año

\section{c. Validación}

La validación es el proceso continuo que nos indica los beneficios y limitaciones de la adaptación protésica concreta. El objetivo es que el audioprotesista se asegure de que el niño recibe la señal óptima, tanto de su propia emisión, como de las demás personas. Implica tanto la supervisión del audífono, como el comportamiento del niño durante su uso.

El audioprotesista y el logopeda pueden comparar los niveles del audífono en diferentes situaciones de audición. Con el empleo de los exámenes sonda-micrófono se obtiene información cuantitativa y objetiva del nivel percibido del habla por el dispositivo auditivo. También es posible comparar la audibilidad con y sin el audífono.

La valoración funcional ha de tener en cuenta la edad auditiva del niño, tomando como referencia para el cálculo de ésta no sólo los meses transcurridos desde la primera adaptación, sino también las horas de uso efectivo de las prótesis.

\subsection{Recomendaciones CODEPEH en relación con la valoración audiológica y la adaptación protésica}

- Los procesos de diagnóstico audiológico y habilitación auditiva tienen la misma prioridad y necesitan desarrollarse en los primeros meses de vida para maximizar el desarrollo óptimo del niño. Esos dos procesos comienzan de forma secuencial, pero se deben desarrollar simultáneamente. 
- Los periodos de seguimiento deben ser:

- los primeros I 8 meses de vida, continuos a demanda de cada situación

- de los I8 meses a los 3 años, cada 3 meses

- de los 3 a los 6 años, cada 6 meses

- mayores de 6 años con sorderas estables, cada año

- El médico especialista en ORL debe de ser el encargado de coordinar las actuaciones del equipo multidisciplinar implicado en la adaptación audioprotésica, junto con el audioprotesista y el logopeda, siendo el responsable de la indicación del tratamiento audioprotésico.

- Es necesario también la formación de grupos de trabajo, organizados según la estructura de cada comunidad autónoma, para el diagnóstico y el tratamiento integral de los niños con sordera.

- Involucrar a los padres en el proceso de tratamiento de los niños con déficit auditivo resulta esencial, para ello, en todo el proceso, se les debe dar una información amplia, comprensible y veraz, que ajuste las expectativas sobre el pronóstico.

- Complementar los resultados de los potenciales evocados auditivos de tronco cerebral (PEATC) con la realización de potenciales evocados de estado estable (PEAEE).

- Los resultados obtenidos en los potenciales evocados auditivos de tronco cerebral y los de estado estable han de ser completados y confirmados mediante audiometría conductual adecuada a la edad del niño.

- Para llevar a cabo una timpanometría en niños menores de 4 meses de edad, se recomienda la utilización de sondas de alta frecuencia $(\mathrm{I} 000 \mathrm{~Hz})$.

- La decisión de proceder a una adaptación protésica debe estar basada en datos audiológicos, del desarrollo del lenguaje y del entorno familiar, escolar y social del niño.

- Hasta los 10-12 años de edad el audífono convencional debe ser retroauricular. Se debe disponer de distintos tipos de moldes para conseguir una correcta adaptación del molde al conducto auditivo externo, sobre todo en lactantes.

- Las prótesis con micrófonos omnidireccionales, por estar siempre en la posición adecuada para recibir el sonido, son más adecuadas en el caso de los lactantes, que suelen gatear.

- Se debería realizar una adaptación binaural siempre, salvo que se observen en el niño conductas que hagan pensar que la adaptación en el peor oído produce un perjuicio en su rendimiento global.

- Individualizar cada caso ya que, entre otros motivos, puede haber niños en los que un mal rendimiento audioprotésico se pueda explicar por la presencia de una neuropatía auditiva o porque presenta lesiones centrales de las áreas de la audición.

\section{PUNTOS CLAVE}

- Antes de proceder a la adaptación audioprotésica, es necesario completar la información audiológica, no obstante puede comenzar la adaptación con estimaciones fiables en un número suficiente de frecuencias

- Los métodos empleados para la valoración audiológica varían en función de la edad y de la adquisición de diferentes habilidades y capacidades para la participación en la evaluación 
- La predicción de los umbrales utilizando los potenciales evocados auditivos de estado estable (PEAEE) se ha comprobado que es suficientemente fiable para estimar los resultados de la audiometría conductual

- La hipoacusia provoca modificaciones en el sistema auditivo central que pueden revertir si se lleva a cabo una adaptación protésica temprana (“aclimatación auditiva”)

- El diagnóstico y tratamiento de la hipoacusia en niños es multidisciplinar. Es indispensable la comunicación continuada y fluida entre el ORL Infantil, el audioprotesista, los logopedas, los educadores y otras personas involucradas supone la presencia de la sordera en uno de sus miembros

Todo ello es además plenamente coincidente con lo hallado en el Estudio sobre Necesidades, Demandas y Expectativas de las Familias de niños y jóvenes con discapacidad auditiva, menores de I 8 años, realizado entre 2004/05 (Jáudenes, 2006) y contrastado después en el Análisis Prospectivo de las Demandas de las Familias atendidas a través de la Red FIAPAS (Jáudenes y Ruíz, 20I I). En ambos trabajos quedó patente que las familias demandan:

- realización de pruebas diagnósticas al nacer

- existencia de equipos especializados: calidad de la atención prestada y de la formación de los profesionales

- acceso a información asequible y derivación a atención temprana logopedia y servicios de orientación a familias

- coordinación interdisciplinar en el seguimiento

- inversión en avances médicos y técnicos, en educación y en el apoyo a las familias

\subsection{Contexto y principios de actuación}

Los documentos más relevantes de las tres últimas décadas ponen de relieve la evolución desde un concepto de intervención centrado en el niño, a otro más amplio y global que concierne al niño, a su familia y a su entorno. Evolución acorde con el progreso actitudinal y social que se ha dado también entorno a la discapacidad en general (Agencia Europea para el Desarrollo de la Educación Especial, 2005) (CERMI, 2005).

En este escenario debemos introducir la Convención Internacional sobre Derechos de las Personas con Discapacidad (2006), normativa internacional vinculante y ratificada por España, plenamente vigente desde 2008 , que viene a subrayar todo lo que desde otro modelo, menos interactivo y menos social, ya anticipaba la legislación española aún de forma insuficiente.

La Atención Temprana, por tanto, se incardina y se ve afectada en mayor o menor medida por 
un amplio marco legislativo generado en España desde el inicio de la década de los ochenta, tanto en materia sanitaria como educativa y de servicios sociales. Pero la gestión de los derechos de las personas con discapacidad no está exenta de cierta complejidad ya que el diagnóstico, la atención temprana, las ayudas técnicas, etc. deben responder a una coordinación muy eficaz, muy eficiente y muy exquisita que empieza en el espacio sociosanitario, pero finalmente termina en el educativo (Jáudenes, 20I4).

\section{Definición, objetivos y principios de la Atención Temprana}

Tras casi quince años de la publicación del Libro Blanco de la Atención Temprana (GAT, 2000) sigue sin existir una norma básica estatal sobre esta materia, al mismo tiempo que el desarrollo normativo de las distintas comunidades autónomas es desigual, tanto por lo que respecta a previsiones y preceptos, como en la aplicación de recursos y financiación.

El Libro Blanco de la Atención Temprana (GAT, 2000) aportó el marco de referencia necesario y generó el suficiente consenso técnico, evolucionando desde el concepto de la "estimulación precoz" a la "atención temprana”, y concretó que ésta es "El conjunto de intervenciones dirigidas a la población infantil de o-6 años, a la familia y al entorno, que tienen por objetivo dar respuesta lo más pronto posible a las necesidades transitorias o permanentes que presentan los niños con trastornos en su desarrollo o que tienen riesgo de padecerlos". Se apuntaba además que “(...) estas intervenciones deben considerar la globalidad del niño, y han de ser planificadas por un equipo profesional de orientación interdisciplinar".

Por otra parte, "la concepción moderna de la atención temprana hace necesario que dispongamos de modelos diagnósticos integradores que consideren, además de las patologías de la salud, los aspectos evolutivos, de aprendizaje, y aquellos otros factores contextuales emocionales y ambientales que inciden en el crecimiento, la maduración y el desarrollo del niño". A lo que se añade el hecho de que la intervención temprana no solo está indicada cuando la deficiencia ya está presente, sino también en los casos en riesgo de padecerla, lo que implica la vigilancia de los factores de riesgo que puedan provocarla (GAT, 2004-20II).

De acuerdo con el Libro Blanco de la Atención Temprana (GAT, 2000), son objetivos propios de esta atención:

- Reducir los efectos de una deficiencia o déficit sobre el conjunto global del desarrollo del niño

- Optimizar, en la medida de lo posible, el curso del desarrollo del niño

- Introducir los mecanismos necesarios de compensación, de eliminación de barreras y para la adaptación a necesidades específicas

- Evitar o reducir la aparición de efectos o déficits secundarios o asociados producidos por un trastorno o situación de alto riesgo

- Atender y cubrir las necesidades y demandas de la familia y del entorno en el que vive el niño

- Considerar al niño como sujeto activo de la intervención

Y entre los principios básicos se definen:

- el diálogo, la integración y la participación en colaboración con la familia

- la gratuidad, universalidad e igualdad de oportunidades, así como la responsabilidad pública

- la interdisciplinariedad y alta cualificación profesional

- la coordinación y planificación de las actuaciones

- la descentralización y la sectorización

Por su parte, la Agencia Europea para el Desarrollo de la Educación del Alumnado con Necesidades Educativas Especiales (20IO) apunta tres prioridades en relación con la Atención Temprana:

- Llegar a toda la población que necesite atención temprana y apoyo, y hacerlo lo antes posible 
- Garantizar la calidad de la oferta dentro de unos estándares de calidad, bien definidos y evaluables, homogéneos en todo el Estado, sin que las diferencias territoriales signifiquen un handicap añadido

- Respetar los derechos de los niños y las niñas y sus familias configurando servicios responsables, centrados en la familia

Del análisis realizado por la Agencia Europea se desprenden, entre otras, propuestas relativas a la necesidad de disponer no sólo de legislación sobre la materia, sino de que ésta se acompañe de la aplicación y seguimiento de medidas políticas. Así mismo, se señala el rol fundamental de los profesionales en todos los niveles, especialmente en el tratamiento de la primera noticia y, en general, respecto a la transmisión de toda la información a las familias por lo que se subraya, además, la importancia de los programas de formación dirigidos a la capacitación para el trabajo en equipos multiprofesionales, compartiendo criterios comunes, objetivos y trabajo eficaz con las familias. Y se incide también en la importancia de la coordinación intersectorial, particularmente en momentos clave de transición cruciales para las familias y sus hijos.

\subsection{Sordera infantil y Atención Temprana}

Sobre el diagnóstico precoz de la sordera se ha avanzado notablemente bajo este cambio de paradigma antes descrito, donde más allá de los aspectos clínicos, se tienen en cuenta tanto el apoyo a las familias como el proceso habilitador audioprotésico y logopédico, incorporados como parte del todo que es el tratamiento integral a los niños y niñas con sordera.

El Programa de Detección Precoz de la Sordera Infantil, aprobado por el Ministerio de Sanidad y las comunidades autónomas, en 2003, es en este aspecto el más avanzado de los países de nuestro entorno. Y, hoy, podemos decir que resulta plenamente concordante con la concepción contemporánea de la Atención Temprana y el modelo social que propugna la
Convención Internacional sobre los Derechos de las Personas con Discapacidad.

\section{Itinerario de derivación e interdisciplinariedad}

La discapacidad auditiva es un claro ejemplo de cómo lo específico de cada sector administrativo que interviene en estas edades (Sanidad, Servicios Sociales y Educación), junto a los diferentes profesionales (pediatra, médico ORL, audioprotesista, logopeda, maestro...) se han de encontrar y, desde un enfoque interdisciplinar e integral, intervenir en torno al niño y su familia en una acción conjunta planificada, coordinada y convergente de recursos, prestaciones y servicios. De un lado, todo lo relacionado con la salud, como la detección y el diagnóstico precoz, el tratamiento médico, la adaptación de prótesis auditivas (audífonos e implantes), y la (re) habilitación médico-funcional. De otro, y sobre la base de lo anterior, la intervención educativa y social: apoyo a las familias, atención temprana, logopedia y apoyo escolar, valoración de la discapacidad, recursos de apoyo, prestaciones y ayudas sanitarias, educativas y sociales (Jáudenes, 20I4).

Todo ello podrá estar al alcance de los niños y niñas sordos y de sus familias si -y solo si- el Diagnóstico Precoz se sigue de una Atención Temprana Especializada (incluida la adaptación protésica y la intervención logopédica) y del Apoyo a las Familias, siempre a través de redes de servicios y profesionales especializados.

Para lograr este objetivo es fundamental que, tal como contempla el programa aprobado en 2003 entre el Ministerio de Sanidad y las comunidades autónomas, las administraciones, en coordinación interdisciplinar a través de unidades de referencia, aseguren ese itinerario de atención y la continuidad del proceso (CODEPEH, 2OIO).

Según el Informe de la Agencia Europea para el Desarrollo de las Necesidades Educativas Especiales (2003/04), las políticas en relación con la atención y apoyo a las personas con discapacidad, en los países de nuestro entorno 
europeo, se planifican sobre cuatro consensos básicos:

- actuar tan pronto como sea posible

- asegurar la continuidad del proceso

- evitar la descoordinación entre servicios y prestaciones

- prevenir el desconcierto y el peregrinar de las familias

De lo expuesto se desprende la relevancia de disponer de un itinerario fácilmente reconocible por todos los implicados y que, en coordinación intersectorial, por lo que respecta a las administraciones, e interdisciplinar, por lo que respecta a los profesionales, pueda dar una respuesta coherente, adecuada y suficiente a las necesidades y a las demandas de los niños con sordera y de sus familias. En este sentido, tenemos que hacer hincapié en cómo hoy ya nadie discute el rol científico que desempeña la familia, sino que este rol se reconoce de forma unívoca y es estudiado con interés ya que no es posible entender ni atender un caso sin prestar la atención debida a la familia que hay detrás. Así, se asume que la atención, la orientación y el apoyo a la familia forma parte del tratamiento y la atención del niño con discapacidad (Jáudenes, I998, 2003) (Jáudenes y Patiño, 2007).

\subsection{Recomendaciones CODEPEH en relación} con la atención temprana al niño sordo y su familia

- La regulación y la universalización de la atención temprana, estableciendo los 0-6 años de edad como el periodo objeto de atención.

- La necesaria coordinación interadministrativa e intersectorial, que responda a una planificación conjunta de servicios y prestaciones dirigidas al niño y a su familia; además de la coherencia de los apoyos y servicios con respecto a las necesidades del niño/a, su trayectoria y su evolución, así como a la toma de decisiones de las familias.
- La pluralidad de respuestas en la atención a la diversidad: no existe un único patrón de respuesta, hay que adaptarse a la variabilidad individual y a la evolución de los tiempos en todos los terrenos: social, tecnológico, legislativo...

- La actualización competencial y la formación especializada de los profesionales de los distintos ámbitos que convergen en la atención al niño sordo y a su familia.

- La incorporación de las familias en los sistemas que les conciernen en relación con sus hijos sordos y su implicación en cada nivel de intervención, incrementando el conocimiento sobre el apoyo familiar entre todos los agentes implicados.

- El establecimiento de circuitos de derivación e itinerarios de atención al niño/a y a su familia, fácilmente identificables y coordinados entre sí, que aseguren la continuidad del proceso.

- La participación del movimiento asociativo de familias, que no sólo actúa como agente y red social, desarrollando programas de apoyo familiar, incluidos grupos de ayuda mutua, sino que contribuye a evitar desigualdades territoriales, aporta asesoramiento técnico especializado e impulsa cambios necesarios.

- La creación de un registro común básico de los resultados de la aplicación del Programa de Detección Precoz de la Sordera Infantil, alimentado con los datos de las distintas comunidades autónomas. Fundamental para estudios epidemiológicos, adopción de medidas de prevención, planificación de recursos sociosanitarios y educativos subsidiarios a las necesidades de la población afectada, posibilitar estudios comparativos a nivel nacional e internacional...

- El establecimiento de consensos científicos, terminológicos, procedimentales, técnicos, educativos, etc. que permitan 
una descripción y clasificación de las necesidades existentes entre la población infantil afectada por problemas auditivos presentes o en riesgo de padecerlos.

- La información y sensibilización social, y entre los agentes implicados, acerca de la trascendencia de la detección y diagnóstico precoz, del seguimiento y vigilancia de los factores de riesgo, de la necesidad de actuar lo antes posible para instaurar el tratamiento médico y audioprotésico, junto a la intervención logopédica temprana.

- La evaluación continua y rigurosa de servicios, recursos, procedimientos y resultados, con medición cuantitativa y cualitativa respecto de todo ello y a través de indicadores de eficacia y de éxito no sólo sobre el nivel de intervención clínico, sino también sobre el progreso del niño, la satisfacción de las familias y de los profesionales, así como sobre los beneficios del entorno.

\section{PUNTOS CLAVE}

- Regulación y universalización de la Atención Temprana, estableciendo los o-6 años de edad como el periodo objeto de atención

- Coordinación interadministrativa e intersectorial, potenciando el trabajo interdisciplinar

- Actualización competencial y formación especializada de todos los profesionales involucrados

- Incorporación de las familias en los sistemas que les conciernen en relación con sus hijos sordos y su implicación en cada nivel de intervención
- Creación de un registro común básico de los resultados de la aplicación del Programa de Detección Precoz de la Sordera Infantil

- Establecimiento de circuitos de derivación e itinerarios de atención al niño sordo y a su familia

- Gestión de resultados a través de una evaluación continua

\section{Anexo de tablas y figuras}

Tabla 1. Causas de Hipoacusia Transmisiva

\begin{tabular}{|c|c|}
\hline OIDO EXTERNO & OIDO MEDIO \\
\hline congénita & congénita \\
\hline infección & infección \\
\hline traumatismo & perforación timpánica \\
\hline obstrucción & tumores \\
\hline \multirow{2}{*}{} & otoesclerosis \\
\cline { 2 - 2 } & traumatismo \\
\cline { 2 - 2 }
\end{tabular}

Tabla 2. Causas de Hipoacusia Neurosensorial

\section{a. CONGÉNITAS}

\begin{tabular}{|l|l|}
\hline HEREDITARIA & NO HEREDITARIA \\
\hline
\end{tabular}

\section{b. ADQUIRIDAS}

\begin{tabular}{|c|}
\hline Prematuridad \\
\hline Hiperbilirruminemia \\
\hline ECMO \\
\hline Hipoxia/Asfixia neonatal \\
\hline $\begin{array}{c}\text { Hemorragia interventricular grados 3-4, } \\
\text { Leucomalacia periventricular }\end{array}$ \\
\hline
\end{tabular}




\begin{tabular}{|c|}
\hline Infección \\
\hline Drogas Ototóxicas \\
\hline Exposición al ruido \\
\hline Traumatismos \\
\hline Tumores \\
\hline $\begin{array}{c}\text { Síndromes neurodegenerativos } \\
\text { (Charcot Marie, Ataxia de Friedrich) }\end{array}$ \\
\hline Intoxicación con metales pesados \\
\hline
\end{tabular}

\section{Tabla 3. Causas de Hipoacusias Centrales}

\begin{tabular}{|c|c|c|c|}
\hline \multirow[b]{2}{*}{$\begin{array}{l}\text { NEUROPATÍA } \\
\text { IDIOPÁTICA (30\%) }\end{array}$} & \multirow[b]{2}{*}{$\begin{array}{c}\text { NEUROPATÍA ADQUIRIDA } \\
\text { (30\%) }\end{array}$} & \multicolumn{2}{|c|}{ NEUROPATÍA GENÉTICA } \\
\hline & & $\begin{array}{c}\text { ASOCIADAS } \\
\text { A PROCESOS } \\
\text { SENSITIVO-MOTORES }\end{array}$ & $\begin{array}{l}\text { NO ASOCIADAS } \\
\text { A OTROS } \\
\text { PROCESOS }\end{array}$ \\
\hline & $\begin{array}{c}\text { Hiperbilirrubinemia con } \\
\text { exanguinotransfusión, } \\
50 \% \\
\text { (Shapiro et ál., 2001) }\end{array}$ & $\begin{array}{c}\text { Enfermedad de Charcot- } \\
\text { Marie-Tooth } \\
\text { (Kovach et ál., 2002) }\end{array}$ & \multirow[t]{5}{*}{$\begin{array}{l}\text { Mutación Q829X } \\
\text { en el gen OTOF }\end{array}$} \\
\hline & $\begin{array}{c}\text { Causas infecciosas, } 10 \% \\
\text { (Race, 2005) }\end{array}$ & Ataxia de Friedrich & \\
\hline & Prematuridad & S Ehrlers Danlos & \\
\hline & Hipoxia neonatal & $\begin{array}{l}\text { Enfermedad Refsun } \\
\text { (Oysy et ál., 2001) }\end{array}$ & \\
\hline & & $\begin{array}{l}\text { Eritroqueratodermia } \\
\text { (López-Bigas et ál., 2001) }\end{array}$ & \\
\hline
\end{tabular}

\section{Tabla 4. Factores de Riesgo de Hipoacusia Infantil}

\begin{tabular}{|c|c|}
\hline Sospecha familiar de sordera* & Meningitis bacteriana* \\
\hline Historia familiar de sordera & Síndromes con sordera* \\
\hline Hipotiroidismo & Enfermedades neurodegenerativas* \\
\hline Estancia en UCIN mayor de 5 días & Anomalías craneofaciales* \\
\hline Exposición a ototóxicos & Ventilación con membrana extracorpórea* \\
\hline Ventilación asistida & Trauma craneal grave* \\
\hline Otitis persistente & Hiperbilirrubinemia* con exanguinotransfusión \\
\hline $\begin{array}{l}\text { Infecciones perinatales* (CMV, herpes, rubeola, } \\
\text { sífilis y toxoplasmosis) }\end{array}$ & Quimioterapia \\
\hline
\end{tabular}

(") factor de muy alto riesgo de sordera postnatal 
Figura 1. Pautas para detectar problemas de audición en la infancia

\section{REVISIÓN PEDIÁTRICA}

El pediatra verificará que se han realizado y superado las pruebas de cribado, de acuerdo con el Programa de Detección Precoz de la Sordera Infantil, así como la existencia de factores de riesgo

1. ¿Gira la cabeza hacia la voz o un sonido?

2. ¿Responde con sonidos vocálicos cuando se le habla?

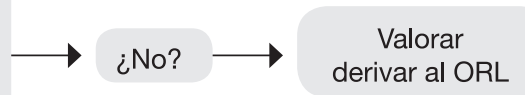

6 meses

3. ¿Reacciona a su nombre?

1. ¿Localiza la fuente del sonido?

2. ¿Señala objetos y personas familiares cuando se le nombran?

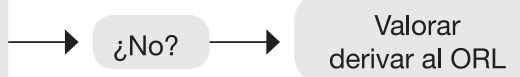

12 meses

3. ¿Dice papá/mamá?

1. ¿Señala partes del cuerpo cuando se le pregunta?

2. ¿Presta atención a las canciones infantiles?

3. ¿Hace frases de dos palabras?

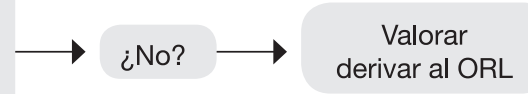

1. ¿Comprende ordenes sencillas sin apoyo de gestos?

24 meses

2. ¿Acude cuando se le llama desde otra

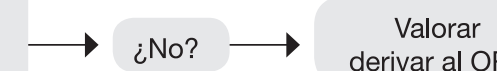
habitación? derivar al ORL

3. ¿Utiliza los pronombres mío/yo/tú?

1. ¿Repite frases sin apoyo visual?

2. ¿Mantiene una conversación?

3. ¿Cuenta una serie de números sencillos (por

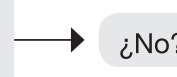

Valorar ej.: del 1 al 10)? 
Referencias bibliográficas

AAP (2007): “Committee on Environmental Health". Pediatrics; IoO: 724-727.

Agencia Europea para el Desarrollo de las Necesidades Educativas Especiales (2005). Atención Temprana. Análisis de la situación en Europa. Aspectos clave y Recomendaciones. Informe Resumen. Bruselas: Agencia Europea para el Desarrollo de las Necesidades Educativas Especiales.

Agencia Europea para el Desarrollo de la Educación del Alumnado con Necesidades Educativas Especiales (2010): Atención Temprana. Progresos y desarrollo. 2005-20I0. Odense, Dinamarca: Agencia Europea para el Desarrollo de la Educación del Alumnado con Necesidades Educativas Especiales. [s.1.]

Alford, R. L. et al. (2OI4): “American College of Medical Genetics and Genomics guideline for the clinical evaluation and etiologic diagnosis of hearing loss". Genet Med; I6(4): 347-55.

American Academy of Pediatrics (r999): “Task force on new born and infant hearing. Newborn and infant hearing loss: detection and intervention". Pediatrics; I03 (527-530).

Aoyagi et al. (I999): "Reliability of $80-\mathrm{Hz}$ amplitude-modulation-following response detected by phase coherence". Audiology o Neuro-otology, 4, 28-37.

Arlinger, S. et al. (1996): "Report of the Eriksholm Workshop on auditory deprivation and acclimatization”. Ear Hear; I7 (3 Suppl): 87S-98S.

Balfour et al. (1998): "Distortion product otoacoustic emission and auditory brainstem response measures of pediatric sensorineural hearing loss with islands of normal sensitivity". Ear and Hearing; I9: 463-472.

Ben-Itzhak, D. et al. (20I4): "Parent Report of the Development of Auditory Skills in Infants and Toddlers Who Use Hearing Aids”. Ear Hear, [Epub ahead of print].
Benito Orejas, J. I. et al. (2013): "Hipoacusia: identificación e intervención precoces”. Pediatría Integral; XVII (5): 330-342.

Briggs L. et al. (20I I): "Outcomes of conventional amplification for pediatric unilateral hearing loss". Ann Otol Rhinol Laryngol 20I I; I 20: 448-54.

Carianne, M. et al. (20I3): "Supplement to the JCIH 2007 Position Statement: principles and guidelines for Early Intervention after a confirmation that a child is deaf or hard-ofhearing. Best Practice Guidelines". Pediatrics; 2OI3-I3I.

CERMI (2005): Plan de Acción del CERMI Estatal en materia de Atención Temprana a Personas con Discapacidad. Madrid: CERMI. Colección CERMI.es, $\mathrm{n}^{\circ} 20$.

Chen, G. et al. (2013): "Screening of delayed-onset hearing loss in preschool children in the midsouth of China". Int J Audiol; 52(8):568-7I.

Chien, W. W. et al. (20I4): "Gene Therapy for Sensorineural Hearing Loss”. Ear Hear. doi: IO.I097 [Epub ahead of print].

Christensen L. et al. (2010): "Update on boneanchored hearing aids in pediatric patients with profound unilateral sensorineural hearing loss". Arch Otolaryngol Head Neck Surg 2010; I36: I75-7.

Coats, A., y Martin, J. (I977): "Human auditory nerve action potentials and brainstem evoked responses: Effects of audiogram shape and lesion". Archives of Otolaryngology $\mathrm{IO}_{3}$, $605-622$.

CODEPEH (Marco et al.) (2004): Control de calidad de los programas de detección precoz de la sordera infantil. Acta Otorrinolaringol Esp 2004, 55 .

CODEPEH (Trinidad et al.) (20I0): Recomendaciones 20Iо CODEPEH. Acta Otorrinolaringol 20I0, En-Febr. 6I(I): 69-77. 
Cohen, L. T. et al. (I99I): “A comparison of steady-state-evoked potentials to modulated tones in awake and sleeping humans". Journal of the Acoustical Society of America; 90: 2467-2479.

Cone-Wesson, B. et al. (2002): "The auditory steady-state response: Comparison with the auditory brainstem response". Journal of the American Academy of Audiology; I3:I73-I 87.

D’Agostino, J. A. et al. (2004): “Auditory neuropathy: A potentially under-recognized neonatal intensive care unit sequela". Advances in Neonatal Care; 344-353.

Dimitrijevic, A. et al. (2002): "Estimating the audiogram using multiple auditory steady-state responses". Journal of the American Academy of Audiology; I3: 205-224.

Federación Estatal de Asociaciones de Profesionales de Atención Temprana - GAT (2005): Recomendaciones técnicas para el desarrollo de la Atención Temprana. Madrid, Real Patronato sobre Discapacidad.

Federación Estatal de Asociaciones de Profesionales de Atención Temprana GAT (20II): Organización Diagnóstica para la Atención Temprana (2004-20II). Madrid, Real Patronato sobre Discapacidad.

Felton, M. et al. (20I4): "The role of stability measurements of the Baha ${ }^{\circledR}$ system in children”. Int J Pediatr Otorhinolaryngol; 78: 5 I3-6.

FIAPAS (Jáudenes, C. y col.) (2003): Guía de Buenas Prácticas para el funcionamiento de los Servicios de Atención y Apoyo a Familias. Madrid, FIAPAS.

FIAPAS (Jáudenes et al.). (2005). Dossier de prevención y atención precoz de los problemas auditivos en edad escolar. Madrid, FIAPAS.

FIAPAS (Jáudenes, C. y Patiño, I.) (2007): Dossier divulgativo para familias con hijos con discapacidad auditiva. Información básica para el acceso temprano a la lengua oral ( $2^{\text {a }}$ edición), Madrid, FIAPAS (2008).

Finckh-Kramer, U. et al. (2000): "Hearing screening of high risk newborn infants". HNS; 48: 2 I 5-20.
Fjermedal, O. y Lazuli, E. (1989): “Pediatric auditory brainstem response and puretone audiometry: Threshold comparison". Audiology; I8:I05-III.

Fligor, B. J. et al. (2005): "Factors associated with sensorineural hearing loss among survivors of extracorporeal membrane oxygenation therapy". Pediatrics; II 5: I 5I9-I 528.

Foulon, I. et al. (2008): “A ro-year prospective study of sensorineural hearing loss in children with congenital cytomegalovirus infection". $J$ Pediatr; I 53(I):84-8.

Fuchsmann C. et al. (2010): "Hearing rehabilitation in congenital aural atresia using the bone-anchored hearing aid: audiological and satisfaction results". Acta Otolaryngol; I30: I343-5I.

Gelfand S. A. et al. (I987): "Long-term effects of monaural, binaural and no amplification in subjects with bilateral hearing loss". Scand Audiol; I6: 20I-7.

Georgalas, C. et al. (2008): “Screening for hearing loss and middle-ear effusion in school-age children, using transient evoked otoacoustic emissions: a feasibility study".J Laryngol Otol; I 22(I 2):I 299-304.

Gorga, M. P. et al. (I985): “Auditory brainstem responses in a case of high-frequency hearing loss". Journal of Speech and Hearing Disorders; 50, 346-350.

Grupo de Atención Temprana (2000): Libro Blanco de la Atención Temprana. Madrid: Real Patronato de Prevención y de Atención a Personas con Minusvalía.

Herdman, A. et al. (2002): "Intracerebral sources of human auditory steady-state responses". Brain Topography; I 5: 69-86.

Hyde, M.L. (2005): "Newborn hearing screening programs: Overview". The Journal of Otolaryngology; 34:S70-S78.

Jáudenes, C. (2006): “Alumnado con discapacidad auditiva: accesibilidad a la comunicación, a la información y al conocimiento" en CNICE (2007), Serie Informes: Accesibilidad, TIC y Educación (on line). Madrid, Centro Nacional 
de Investigación y Comunicación Educativa$\mathrm{M}^{\mathrm{o}}$ de Educación. ‘http//ares.cnice.med informes/I 7 /index.htm>, acceso 8 de septiembre de 2014 .

Jáudenes, C. (2006). “La población con discapacidad auditiva en cifras. Revisión de dos Estudios Sociológicos”. Revista FIAPAS, mayojunio 2006, ${ }^{\circ}$ I IO, Separata. Madrid.

Jáudenes, C. et al. (2007) Estudio sobre la situación educativa del alumnado con discapacidad auditiva. Madrid, FIAPAS.

Jáudenes, C. y Ruíz, E. (20I I) “Análisis prospectivo de demandas atendidas en la Red de Atención y Apoyo a Familias-FIAPAS. Informe Final”. Revista FIAPAS, abril-junio 20I I, n ${ }^{\circ}$ I37. Separata. Madrid.

Jaudenes, C. (20I4): "Las personas sordas y sus familias en la intersección sociosanitaria”. En CERMI.es, febrero 2014 (en línea). http://semanal.cermi.es/noticia/Espaciosociosanitario-discapacidad-opinion-diezarticulos.aspx $<$

Kerkhofs K. y Smith M. (20I3): "Early hearing aid fitting in children: challenges and results". B-ENT; Suppl 2I:I7-25.

Kovach, M. J. et al. (2002): «Anticipation in a unique family with Charcot-Marie-Tooth syndrome and deafness: delineation of the clinical features and review of the literature». Am J Med Gene; 108:295า-303.

Levi, E. C. et al. (I995): "Coherence analysis of envelope following response (Errs) and frequency-following responses (Firs) in infants and adults". Hearing Research; 89: 2I-27.

Lieu J. E. et al. (2013): "Unilateral hearing loss in children: speech-language and school performance”. B-ENT; Suppl 2I: IO7-I 5.

López-Bigas, N. et al. (200I): “Connexin 3 I $\left(\mathrm{GJB}_{3}\right)$ is expressed in the peripheral and auditory nerves and causes neuropathy and hearing impairment". Hum Mol Genet; I0:947-52.

Lü, J. et al. (20I I): "Screening for delayedonset hearing loss in preschool children who previously passed the newborn hearing screening”. Int J. Pediatr Otorhinolaryngol; 75(8): $1045^{-9}$.

Marco, J. y Mateu, S. (coord) (2003): Libro Blanco sobre la Hipoacusia. Detección precoz de la hipoacusia en recién nacidos. CODEPEH y Ministerio de Sanidad y Consumo.

Margolis, R. et al. (2003): "Tympanometry in newborn infants - I kHz norms". Journal of the American Academy of Audiology; I4: 383392.

Martins, F. T. et al. (2013): "Optimization of simultaneous screening of the main mutations involved in non-syndromic deafness using the TaqMan ${ }^{\circledR}$ OpenArray ${ }^{\mathrm{TM}}$ Genotyping platform”. BMC Med Genet; 24; I 4:I I 2.

Mauk, G. W. et al. (I99I): "The effectiveness of screening programs based on high risk characteristics in early identification of hearing impairment”. Ear Hear; I 2: 3 I 2-9.

Mitchell, R. E. y Karmchmer M.A.: "Chasing the mythical ten percent: parental hearing status of deaf and hard of hearing students in the United States", Sign Language Studies. Vol. 4, (2) I38I63. Gallaudet University Press.

Moore, B. C. et al. (2000): “A test for the diagnosis of dead regions in the cochlea". Br J Audiol; 34: 205-24.

Northern, J. y Downs, M. (I99I): "Hearing in children” Baltimore: Lippicott Williams and Wilkins.

Oysu, C. et al. (2002): "Incidence of cochlear involvement in hyper bilirrubinemia deafness". Ann Otorhinolarygol; I I I:IO2 I-5.

Oysy, E. et al. (200I): "The site of the hearing loss in Refsum's disease". Int J Pediatr Otorhinolaryngol; 6I: I29-34.

Paradise, J. et al. (1976): “Tympanometry detection of middle ear effusion in infants and young children". Pediatrics, 58, 198-2 го.

Pérez-Abalo, M. C. et al. (200I): "Steady-state responses to multiple amplitude-modulated tones: An optimized method to test frequencyspecific thresholds in hearing-impaired children and normal hearing subjects". Ear and Hearing; 22: 200-2II. 
Rance, G. (2005): “Auditory Neuropathy/Dyssynchrony and its Perceptual Consequences". Trends Amplification; 9: I-43.

Rance, G. et al. (2005): "Hearing threshold estimation in infants using auditory steady-state responses". Journal of the American Academy of Audiology; г6: 29I-300.

Sand, N. et al. (2005): "Pediatricians reported practices regarding developmental screening: do guidelines work? Do they help?". Pediatrics; II 6:I74-I79.

Shapiro, S. M. et al. (200I): "Bilirubin and the auditory system». J Perinatol; 2I:52-5.

Sheykholeslami, K. et al. (200I): "An isolated and sporadic auditory neuropathy (auditory nerve disease): report of five patients". J Laryngol Otol; I I 5:530-4.

Silvestre, N. y FIAPAS (2008): Estudio Investigación. Interacciones comunicativas entre padres/madres e hijos/as con sordera. Madrid, FIAPAS.

Silvestre, N. y Ramspott, A. (2004). "Valoración del discurso narrativo y de sus precursores en el alumnado con déficit auditivo: Influencia de las modalidades comunicativas". Revista FIAPAS 97. Separata.

Smith, R. J. H. y Gooi, A. (20I4): Hearing impairment in children: Etiology (en línea). http://www.uptodate.com/`, acceso 4 de septiembre de 2014 .

Stapells, D. R. (1989): “Auditory brainstem response assessment of infants and children”. Seminars in Hearing; IO, 229-25I.

Stapells, D. R. et al. (I994): "Electrophysiologic measures of frequency-specific auditory function”. In J. T. Jacobson (Ed.), Principles and application in auditory-evoked potentials (pp. 25I-283). Boston Ed: Allyn and Bacon.
Stapells, D. R., y Oates, P. (I997): "Estimation of the pure-tone audiogram by the auditory brainstem response: A review”. Audiology Neuro-Otology; 2: 225-280.

Swanepoel, D. (2004): “Auditory steady-state response for children with severe-to-profound hearing loss". Archives of Otolaryngology Head and Neck Surgery, I3O, 53 I-535.

Tokgöz-Y1lmaz, S. et al. (2OI3): "Evaluation of hearing and speech-language in preschool children: how important, why we should perform?" Turk J Pediatr; 55(6):606-I I.

Tomblin, J. B. et al. (2OI4): "The influence of hearing aids on the speech and language development of children with hearing loss". JAMA Otolaryngol Head Neck Surg; I40: 403-9.

Torres, S. et al. (1995). Deficiencia auditiva: Aspectos psicoevolutivos y educativos. Málaga: Aljibe.

Torres, S. (1998). "Memoria, fonología y sordera". Revista FIAPAS, 62, 25-28

Trinidad, G. y Jáudenes, C. (coord) (20I I): Sordera Infantil. Del diagnóstico precoz a la inclusión educativa. Guía práctica para el abordaje interdisciplinar. Madrid, FIAPAS

Villalba, A. et al. (2005). La lectura en los sordos prelocutivos. Propuestas para un programa de entrenamiento. Madrid: Entha ediciones.

Watkin, P. et al. (20I2): “The longitudinal follow up of a universal neonatal hearing screen: the implications for confirming deafness in childhood". Int J Audiol; 5 I(7):5 I9-28.

Watkin, P. M. y Baldwin M. (20I I): “Identifying deafness in early childhood: requirements after the newborn hearing screening". Arc Dis Child; 96: 62-66. 